\title{
Combined Hepatocellular-Cholangiocarcinoma with Stem Cell Features-Case Report
}

\author{
Leonardo Verza ${ }^{1}$, Carlos Henrique Rosas ${ }^{1}$, Gabriel Marques Neves ${ }^{1}$, Tércia Neves ${ }^{2}$, \\ Maria Dirlei Begnami², Marcos Duarte Guimarães ${ }^{3}$ \\ ${ }^{1}$ Imaging Department, A.C. Camargo Cancer Center, São Paulo, Brazil \\ ${ }^{2}$ Pathology Department, A.C. Camargo Cancer Center, São Paulo, Brazil \\ ${ }^{3}$ AC Camargo Cancer Center and Universidade Federal do Vale do São Francisco, São Paulo, Brazil \\ Email: *leoverza9@gmail.com
}

How to cite this paper: Verza, L., Rosas, C.H., Neves, G.M., Neves, T., Begnami, M.D. and Guimarães, M.D. (2018) Combined Hepatocellular-Cholangiocarcinoma with Stem Cell Features-Case Report. Case Reports in Clinical Medicine, 7, 526-531.

https://doi.org/10.4236/crcm.2018.710046

Received: August 17, 2018

Accepted: October 26, 2018

Published: October 29, 2018

Copyright $\odot 2018$ by authors and Scientific Research Publishing Inc. This work is licensed under the Creative Commons Attribution International License (CC BY 4.0).

http://creativecommons.org/licenses/by/4.0/

\begin{abstract}
Combined hepatocholangiocarcinoma is a rare and unique form of primary hepatic neoplasm, expressing histopathological and phenotypic aspects of hepatocellularcarcinoma and cholangiocarcinoma in the same tumor. Diagnosis may be performed by imaging, showing typical features of both components. We present a case of a 55-year-old woman presenting with abdominal pain and a hepatic mass. The patient underwent surgery and combined hepatocholangiocarcinoma with stem cells features was confirmed on pathological analysis. There are no signs of recurrence to date. Combined hepatocholangiocarcinoma requires a preoperative diagnosis, since it is a unique entity with higher rates of local and lymph node recurrence, compared to isolated forms.
\end{abstract}

\section{Keywords}

Hepatocellular Carcinoma, Cholangiocarcinoma, Liver Neoplasm, Diagnostic Imaging

\section{Introduction}

Hepatocellular carcinoma (HCC) is an important cancer-related cause of death and accounts for about $80 \%$ of primary liver tumors, with a higher incidence in Asian countries [1]. In Brazil, it's not even between the ten most prevalent neoplasms and there is a strong association with viral hepatitis and cirrhosis [2]. Cholangiocarcinoma (CC) accounts for $5 \%$ of primary hepatic neoplasms and is associated with chronic inflammatory involvement of the biliary tract [3].

Combined hepatocholangiocarcinoma $(\mathrm{CHC})$ is a rare primary liver cancer, 
expressing pathological and phenotypic features of HCC and CC [4]. It was first clearly described in 1949 by Lisa and Allen [5], however, still constitutes an uncertain behavior tumor due to lack of data in the literature and no consensus about its treatment. The mean survival of $\mathrm{CHC}$ after resection is lower compared to HCC or CC alone [1] [6], usually related to local and lymph node recurrence. [6].

Regarding the clinical and epidemiological features of the combined tumor, studies have shown differences between Asian and Western populations [1] [5]. In Asians, there is a strong male predominance, high alpha-fetoprotein (AFP) levels, greater association with $\mathrm{B}$ and $\mathrm{C}$ virus infection and frequent chronic liver disease, simulating a HCC behavior [1] [4] [7]. Conversely, some western studies showed no gender predominance, low relation with viral hepatitis and cirrhosis, simulating closer aspects to CC [1] [3] [5]. The distinction was also noticed on imaging and treatment, since tumor unresectability is based on hepatic dysfunction degree, which was worst in Asian population, likely related to chronic hepatitis. Tumor size at diagnosis was higher in the western group [6].

\section{Case Report}

A 55-year-old female presented a 2 months-history of diffuse and nonspecific abdominal pain. After an initial medical evaluation by an outside clinician, an abdominal ultrasonography was requested and revealed a liver mass. No initial laboratory was requested. Core liver biopsy performed at an outside facility revealed a malignant epithelioid neoplasm. Therefore, the patient was referred to our institution for further evaluation. Physical examination was unremarkable. Laboratory test showed an AFP of $9693 \mathrm{ng} / \mathrm{ml}$ (Reference: up to $8 \mathrm{ng} / \mathrm{ml}$ ) and gamma-glutamyltransferase (GGT) of $84 \mathrm{u} / \mathrm{L}$ (Reference: $36 \mathrm{u} / \mathrm{L}$ ). Serology for hepatitis $\mathrm{B}$ and $\mathrm{C}$ were negative. Abdominal contrast-enhanced computed tomography (CT) revealed a heterogeneous mass (Figure 1 and Figure 2) occupying segments $\mathrm{V}$ and VI, causing hepatic contour irregularity associated to a hepatic hilar node measuring $16 \mathrm{~mm}$ on shortest axis and lack of chronic liver disease. The patient underwent surgical resection of segments VI and VII as well hilar lymphadenectomy. Postoperative pathological analysis confirmed combined hepatocellular-cholangiocarcinoma with stem cells features (Figure 3). The patient underwent four cycles of postoperative adjuvant chemotherapy with Gentabicin and Cisplatin. Afterwards, surveillance with bi-monthly MRI and thoracic CT was proposed. No signs of recurrence in the first six months of surveillance.

\section{Discussion}

$\mathrm{CHC}$ is a unique clinical, pathological and diagnostic entity distinct from pure hepatocellular or cholangiocarcinoma. Classifications proposed by Allen and $L i-$ sa's [5] and Goodman et al. [8] were the first to divided the CHC subtypes. The first, as follows: type A (HCC and CC foci at different sites in the liver), B (adjacent growth foci with a transition area), and C (combined HCC and CC components, originating from the same tumor). Goodman's classification, as follows: 


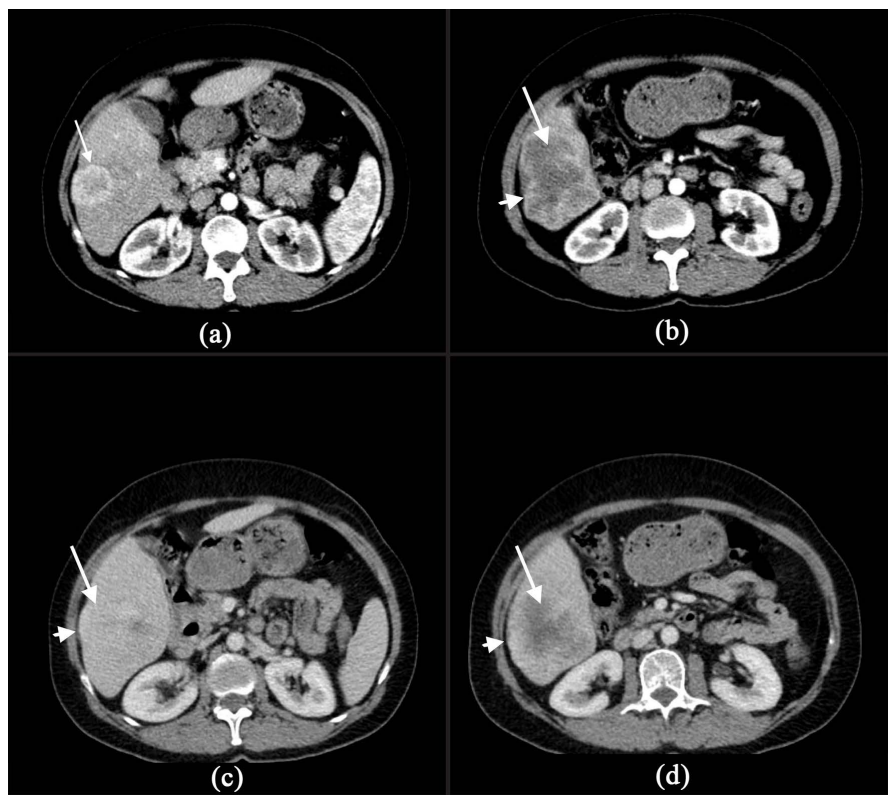

Figure 1. Combined hepatocellular-cholangiocarcinoma on dynamic-CT showing features of HCC ((a)-(c)) and CC ((b)-(d)) components on arterial and portal phases ((a)-(b)) Arterial phase at different lesion levels. (a) Axial at upper level on HCC component. The nodule (arrow) has a strong early and homogeneous enhancement. (b) Axial at a lower level on CC component. Hypodense mass (arrow), with irregular contours and a peripheral rim showing early enhancement (arrowhead). (c) (d) Portal phase at different lesion levels: (c) Axial at HCC component shows the tumour with same density as normal liver parenchyma likely the washout, a typical feature of HCC on dynamic study. Note the hepatic contour irregularity (arrowhead); (d) Axial at CC component, shows a slow and centripetal enhancement, due to fibrous tissue and the hypervascular rim (arrowhead) becomes isodense to normal liver. Those features are very suggestive of cholangiocarcinoma component.

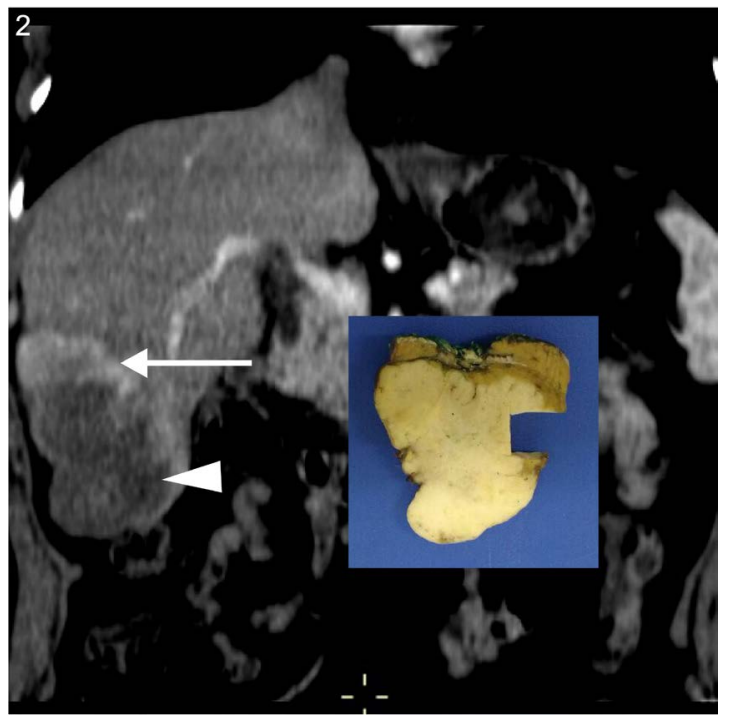

Figure 2. Coronal arterial-phase and the macroscopic tumor on the left after resection. The tumor measures $6 \mathrm{~cm}$ on longest axis. Dynamic CT shows HCC (arrow) and CC components (arrowhead). Unfortunately, is not possible to identify the HCC and CC on macroscopic evaluation. 


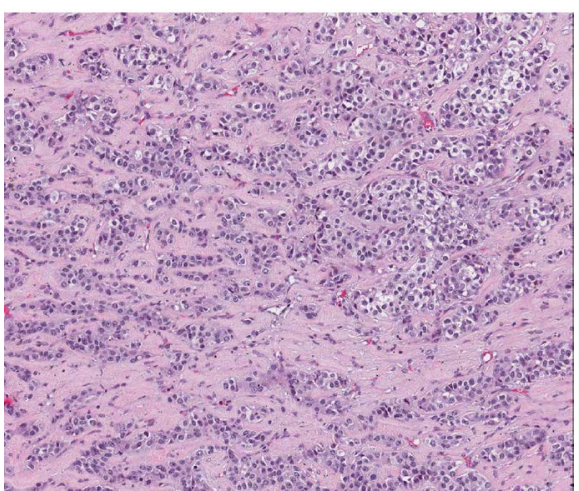

(a)

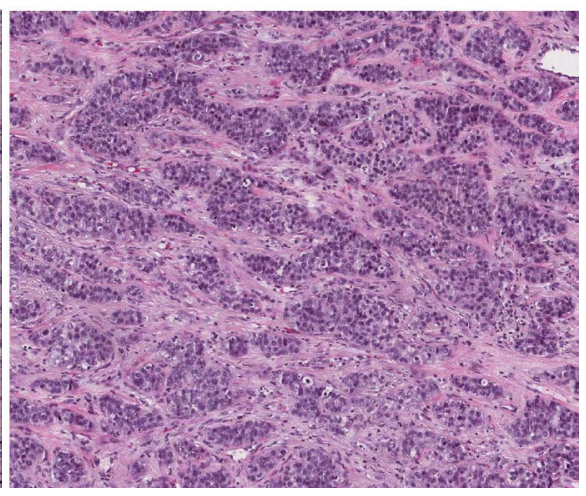

(b)

Figure 3. Hematoxilin and eosin $40 \times$ histological study of combined hepatocellular-cholangiocarcinoma, showing in (a), clear cells of hepatocellular carcinoma and (b), glandular formation representing cholangiocarcinoma.

type I (synchronic or metachronic foci in different sites of the liver that may collide, also known as collision tumor), type 2 (components are mixed and originate from the same tumor) and type 3 (fibrolamellar with mucin-producing pseudoglands). However, along the years' new immunohistochemistry techniques were developed and studies showed that $\mathrm{CHC}$ is even more complex.

The current classification proposed by the World Health Organization (WHO) defines CHC as a tumor with unambiguous findings of combined HCC and CC elements, that subdivides, as follows: classical type; cells expressing typical HCC markers (HepPar1, CD10, rabbit polyclonal, CEA, and AFP) and CC (mucin adenocarcinoma with fibrosis and biliary immunohistochemical markers, as CK type) or stem cells/progenitor cells type, expressing hepatic stem cells specific markers (KIT, CK14, CD34 and CD56, the latter much rare) [9].

Imaging plays an important role ondiagnosis of liver tumors, avoiding unnecessary procedures and biopsies, once mostly primary tumors show typical imaging features [2]. Despite the unique pathologic and phenotypic features, the diagnostic accuracy of CHC is poor [3]. Radiological diagnosis of HCC and CC may be done by $\mathrm{CT}$ and magnetic resonance (MRI) with dynamic study. The histologic composition and relative ratio of $\mathrm{CC}$ and HCC components within $\mathrm{CHC}$ tumors, appears to dictate the imaging appearance [3]. Some typical findings, such as exuberant arterial-enhancement and hypodensity on portal phase, also known as washout, besides the presence of a pseudocapsule, are typical patterns of HCC on dynamic CT [3] [7]. CC usually presents as a hypoattenuating lesion with a slight peripheral and centripetal contrast-enhancing, associated with some biliary dilatation degree [10]. The signal and attenuation may vary according to the fibrosis amount on CC component [2] [3]. LI-RADS is the main algorithm, being used to classify the primary hepatic tumor in HCC or non-HCC based on imaging characteristics. The score is helpful and has a high specificity; however it presents potential pitfalls in depiction of mixed lesions, such as $\mathrm{CHC}[3]$. 
The preoperative diagnosis of $\mathrm{CHC}$ on enhanced $\mathrm{CT}$ would contribute to appropriate management of operative methods. This is especially true in places where HCC and CC is diagnosed on the basis of imaging appearance alone. Local ablative therapies, which have benefit in HCC, and chemotherapy, used in advanced CC, has limited indications for $\mathrm{CHC}$, showing the significance of aggressive surgery and a wide lymph node resection [7].

In current literature, there is no specific algorithm for $\mathrm{CHC}$ diagnosis, based on imaging features alone, as LI-RADS for HCC, for example. However, some studies tried to correlate imaging and pathological findings to depict CHC [2] [11]. The contrast-enhancement pattern, which is typical for each component, is the main feature to be evaluated [2]. Since there are hypovascular and hypervascular areas in the same hepatic mass, the $\mathrm{CHC}$ should be mentioned by the radiologist as a differential diagnosis. In addition, the disagreement between pathological result and imaging, has also been reported as a clue for $\mathrm{CHC}$, since the tissue fragment might represent one tumor component only. Causes of misdiagnosis are atypical behavior of each component and small size [2]. The intermediate tumor cell differentiation degree between HCC and CC can also be reproduced in imaging, showing a mixing of the typical findings of each component [2].

Despite the high AFP levels, that resemble to Asians population studies, our patient had a clinical history similar to those described in case series of $\mathrm{CHC}$ in western population, such as negative history of viral hepatitis, absence of chronic hepatitis and large tumor size on diagnosis [6]. Regarding the imaging features, dynamic CT showed typical features of HCC and CC in the same tumor. The postoperative pathological evaluation, had expression of both components with liver stem cells features. The HCC component represented by thickened trabeculae's composed of polygonal cells with abundant granular and eosinophilic cytoplasm and lack of stromal tissue (Figure 3(a)). The CC component with glandular formation with cuboidal cells and dense fibrotic stroma (Figure 3(b)).

\section{Conclusion}

In conclusion, $\mathrm{CHC}$ is a rare and unique liver primary neoplasm, showing higher rates of recurrence and mortality when compared to isolated forms, according to published literature. Given the rare occurrence and variety of demographic and clinical profiles, the preoperative diagnosis of $\mathrm{CHC}$ remains challenging; however, imaging plays a key role and may be helpful when performed by dynamic CT or MRI.

\section{Conflicts of Interest}

The authors declare no conflicts of interest regarding the publication of this paper.

\section{References}

[1] Ng, I.O., Shek, T.W., Nicholls, J. and Ma, L.T. (1998) Combined Hepatocellu- 
lar-Cholangiocarcinoma: A Clinicopathological Study. Journal of Gastroenterology and Hepatology, 13, 34-40. https://doi.org/10.1111/j.1440-1746.1998.tb00542.x

[2] Nishie, A., Yoshimitsu, K., Asayama, Y., Irie, H., Aibe, H., Tajima, T., et al. (2005) Detection of Combined Hepatocellular and Cholangiocarcinomas on Enhanced CT: Comparison with Histologic Findings. American Journal of Roentgenology, 184, 1157-1162. https://doi.org/10.2214/ajr.184.4.01841157

[3] Potretzke, T.A., Tan, B.R., Doyle, M.B., Brunt, E.M., Heiken, J.P. and Fowler, K.J. (2016) Imaging Features of Biphenotypic Primary Liver Carcinoma (Hepatocholangiocarcinoma) and the Potential to Mimic Hepatocellular Carcinoma: LI-RADS Analysis of CT and MRI Features in 61 Cases. American Journal of Roentgenology, 207, 25-31. https://doi.org/10.2214/AJR.15.14997

[4] Maximin, S., Ganeshan, D.M., Shanbhogue, A.K., Dighe, M.K., Yeh, M.M., Kolokythas, O., et al. (2014) Current Update on Combined Hepatocellular-Cholangiocarcinoma. European Journal of Radiology Open, 1, 40-48.

https://doi.org/10.1016/j.ejro.2014.07.001

[5] Alen, R.A. and Lisa, J.R. (1949) Combined Liver Cell and Bile Duct Carcinoma. American Journal of Pathology, 25, 647-655.

[6] Maeda, T., Adachi, E., Kajiyama, K., Sugimachi, K. and Tsuneyoshi, M. (1995) Combined Hepatocellular and Cholangiocarcinoma: Proposed Criteria According to Cytokeratin Expression and Analysis of Clinicopathologic Features. Human Pathology, 26, 956-964. https://doi.org/10.1016/0046-8177(95)90084-5

[7] Jarnagin, W.R., Weber, S., Tickoo, S.K., Koea, J.B., Obiekwe, S., Fong, Y., et al. (2002) Combined Hepatocellular and Cholangiocarcinoma: Demographic, Clinical, and Prognostic Factors. Cancer, 94, 2040-2046. https://doi.org/10.1002/cncr.10392

[8] Goodman, Z.D., Ishak, K.G., Langloss, J.M., Sesterhenn, I.A. and Rabin, L. (1985) Combined Hepatocellular-Cholangiocarcinoma. A Histologic and Immunohistochemical Study. Cancer, 55, 124-135.

https://doi.org/10.1002/1097-0142(19850101)55:1<124::AID-CNCR2820550120>3.0 .CO;2-Z

[9] Terada, T. (2013) Combined Hepatocellular-Cholangiocarcinoma with Stem Cell Features, Ductal Plate Malformation Subtype: A Case Report and Proposal of a New Subtype. International Journal of Clinical and Experimental Pathology, 6, 737-748.

[10] Maetani, Y., Itoh, K., Watanabe, C., Shibata, T., Ametani, F., Yamabe, H., et al. (2001) MR Imaging of Intrahepatic Cholangiocarcinoma with Pathologic Correlation. American Journal of Roentgenology, 176, 1499-1507. https://doi.org/10.2214/ajr.176.6.1761499

[11] Bhagat, V., Javle, M., Yu, J., Agrawal, A., Gibbs, J.F., Kuvshinoff, B., et al. (2006) Combined Hepatocholangiocarcinoma: Case-Series and Review of Literature. International Journal of Gastrointestinal Cancer, 37, 27-34.

https://doi.org/10.1385/IJGC:37:1:27 